\title{
Role of Cross-Sectional Imaging in Tongue Lesions
}

\author{
BT Deepthi ${ }^{\oplus 1}$, M Gautham ${ }^{\circledR 2}$, Jefrin Roy Mathew ${ }^{\circledR 1}$ \\ ${ }^{1}$ Junior Resident, Department of Radiology, Rajarajeswari Medical College and Hospital, Karnataka, Bangalore, India, ${ }^{2}$ Professor, Department of Radiology, \\ Rajarajeswari Medical College and Hospital, Bangalore, Karnataka, India.
}

\section{Abstract}

Background: The purpose of this study was to determine the role of CT and MR imaging in demonstrating lesions of the tongue. Imaging can help to decide the further management of the patient, and when resection is considered, the precise extent of the lesion can be delineated, and also if organ conservation therapy can be suggested. Hence, knowing the differentiating characteristics of these lesions is essential for a Radiologist to narrow the differential diagnosis. The aim of the study is to describe the imaging findings of various tongue lesions, give radio-pathological correlation, and discuss the role of CT and MRI in planning further appropriate treatment, the extent of involvement of adjacent structures, resectability, postoperative reconstruction \& prognosis. Subjects and Methods: Twenty patients with tongue masses were prospectively evaluated with CT \& MRI for eighteen months from June 2018 - Nov 2019. Contrast-enhanced CT axial images with reconstruction were acquired. MRI plain \& contrast study done. Imaging findings \& diagnoses were later correlated with surgical and histopathological results in all possible cases. Results: Among twenty patients, three patients revealed no abnormality; seventeen patients with findings on imaging include twelve squamous cell carcinoma, two venous malformations, two thyroglossal cysts, one hemangioma \& one fatty lipoma. Conclusion: Few specific lesion characteristics can aid in narrowing the differential diagnosis. Solid high-density lesions in the midline mostly represent lingual thyroids. Calcifications likely indicate goitrous transformation. Phleboliths are highly suggestive of venous malformations. Multinodular, thin-rim enhancing cystic lesions are indicative of lymphatic malformations, primarily when fluid-fluid levels are found. Fat/calcium content within a complex cystic lesion is specific for a dermoid cyst, whereas diffusion restriction within a pure cystic lesion is suggestive of an epidermoid cyst. Finally, when an injury is Trans spatial, three differentials to be considered are highly aggressive malignancies, congenital masses \& aggressive infections.

Keywords: Tongue lesions, Computed tomography, Magnetic Resonance Imaging, contrast-enhanced imaging, squamous cell carcinoma.

Corresponding Author: BT Deepthi, Junior Resident, Department of Radiology, Rajarajeswari Medical College and Hospital, Karnataka, Bangalore, India.

E-mail: bt.deepthi@gmail.com

Received: 30 March 2020

Published: 25 June 2020

\section{Introduction}

Although most of the tongue masses are squamous cell carcinomas, various unusual lesions may also affect the tongue. Most of the congenital lesions found in children are seen at the root of the language. The root of the tongue is relatively resistant to primary neoplastic and infectious processes due to its high percentage of skeletal muscle and lack of significant lymphatic tissue. Lesions involving the root of the tongue can be classified into congenital vascular and nonvascular lesions, infections, and neoplasms. ${ }^{[1]}$ The ageadjusted incidence in India being 20 per 100,000 population, squamous cell cancers (SCC) form the bulk of the lesions involving the tongue. ${ }^{[2]}$ SCC invariably invades the deep tongue from adjacent mucosal surfaces of the oral cavity and anterior oropharynx and does not originate from the deep tongue structures. ${ }^{[3]}$ Imaging provides crucial details for the appropriate management of these lesions. In the present study, we discuss the brief anatomy of tongue, imaging findings in various injuries, and suggest optimal modality \& type of crosssectional investigation for different pathological lesions.

\section{Subjects and Methods}

Twenty patients with clinically suspected tongue masses were prospectively evaluated with CT \& MRI for eighteen months from June 2018 to Nov 2019. Contrast-enhanced axial images with multiplanar reconstruction were acquired using 64 slice Siemens CT machine. MRI plain \& contrast study was obtained with T1, T2, T1 Fat saturated, DWI \& GRE sequences \& multiplanar image acquisition using Siemens Avento $1.5 \mathrm{~T}$ machine. Imaging findings \& diagnoses were 
Table 1: Depicts the percentage of various lesions seen in our patients.

\begin{tabular}{|ll}
\hline Diagnosis & No of patients \\
\hline SCC & 12 \\
\hline Venous malformation & 2 \\
\hline Thyroglossal cyst & 2 \\
\hline Lipoma & 1 \\
\hline Hemangioma & 1 \\
\hline NAD & 2 \\
\hline Total & 20 \\
\hline
\end{tabular}

later correlated with surgical and histopathological results in all possible cases.

CT being a comparatively standard modality of imaging in these lesions has to be combined with contrast study for optimal characterization \& better diagnosis. Puffed cheek technique is of absolute necessity to differentiate buccal \& gingival spaces, and for a better understanding of extension of the lesion. Coronal \& sagittal reconstruction in both bone $\&$ soft tissue windows is of added advantage. MRI is usually reserved for imaging larger lesions, evaluating their extent of involvement and complicated post-surgical cases. Most of the congenital lesions found in children are located at the root of the tongue. MR imaging is used to evaluate these lesions more frequently, due to the lack of exposure to ionizing radiation. Moreover, it provides superior soft-tissue characterization, which is of importance in the evaluation of the suprahyoid neck. Epidermoid cysts may be differentiated from dermoid cysts using Diffusion- weighted imaging, as the former often show restricted diffusion. Sedation may usually be required in children below the age of 7 years, although specific training and the use of audiovisual entertainment systems can significantly reduce this need. ${ }^{[4]}$

\section{Results}

Among twenty patients, two patients had no significant abnormality; eighteen patients with positive findings on imaging included twelve cases of squamous cell carcinomas, two venous malformations, two thyroglossal cysts, one hemangioma $\&$ one fatty lipoma. [Table \& Graph 1].

In the eighteen positive cases, twelve had malignant lesions \& six benign lesions [Table \& Graph 2].

In our study, we observed that congenital lesions were more common in patients In our study, we observed that congenital lesions were more common in patients below 40 years, predominantly in females, while acquired injuries were common in patients above 40 years of age, mostly in males.

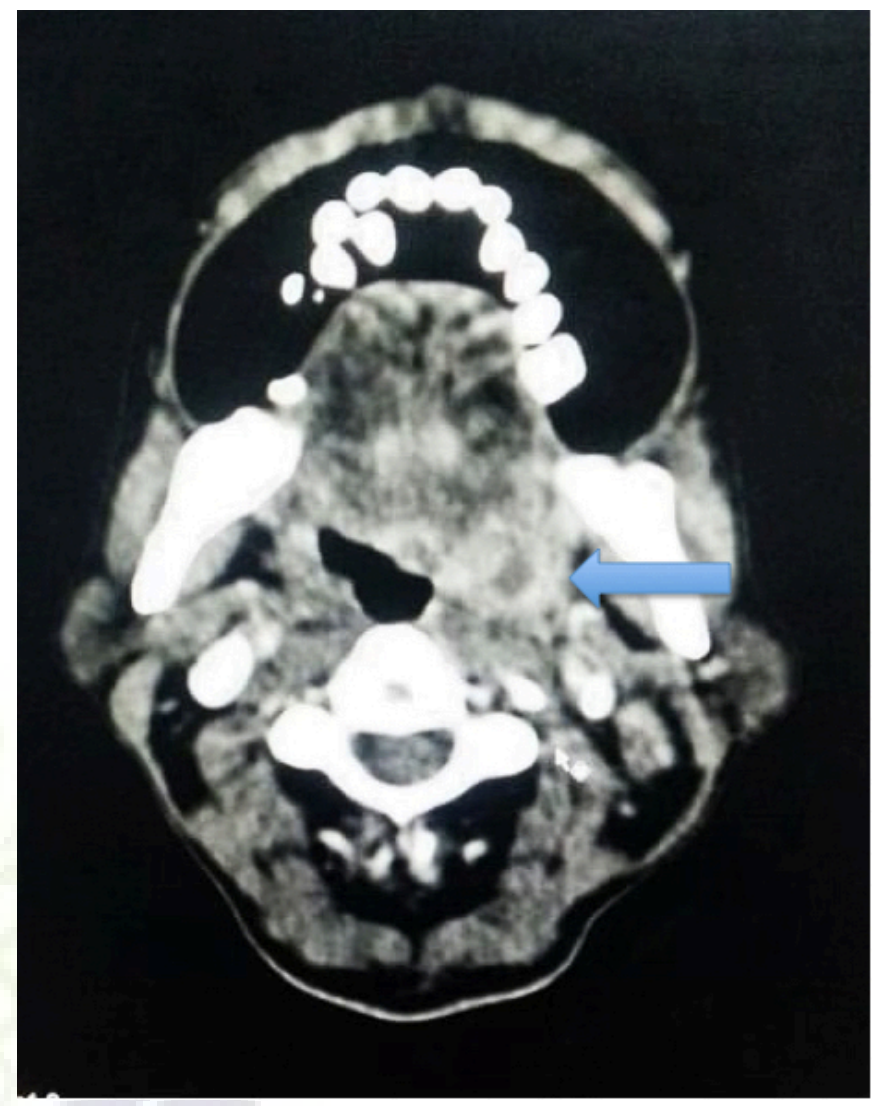

Figure 1: Contrast enhanced axial CT image of a 60 year old male patient shows an ill-defined heterogeneously enhancing solid mass lesion involving the base oftongue and oropharyngeal wall on the left side- Case of Carcinoma tongue.

Table 2: Depicts the percentage of patients with benign and malignant lesions.

\begin{tabular}{ll}
\hline Diagnosis & \\
Malignant & 12 \\
Benign & 6 \\
\hline
\end{tabular}

In patients diagnosed with SCC, eight patients were above 60 years of age, three patients 40-60 years of age, and one patient above 20 years of age. Eight patients showed an association with risk factors like tobacco chewing, smoking, and alcohol.

SCC showed a predilection for males with an incidence being $72 \%$ among males and $28 \%$ among females. The most common site involved in the SCC of tongue in this study was the lateral aspect and anterior two-third of the tongue. In two patients, the lesions showed erosion of hyoid bone. Two patients had lesions that extended across the midline. Four patients of SCC had lesions extending into the retromolar 


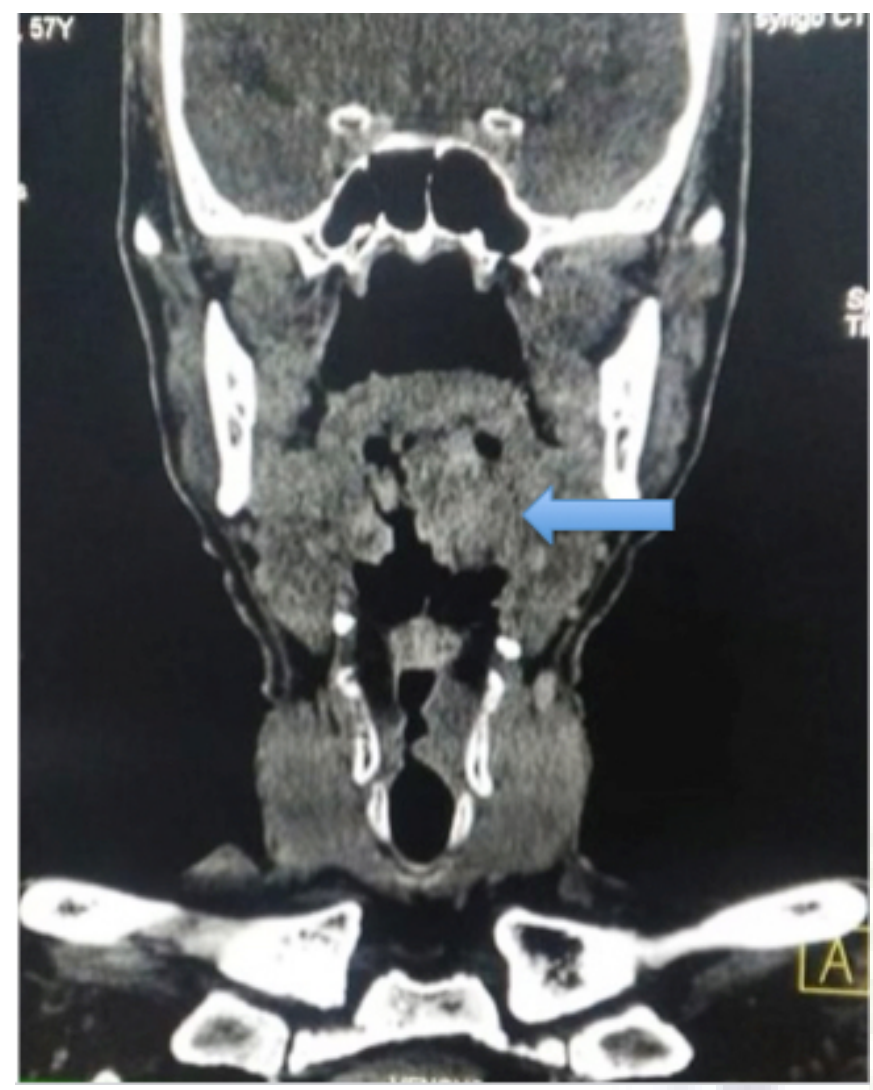

Figure 2: Coronal CT image of a 57 year old male patient shows an ill-definedhypodense solid mass lesion involving the base of tongue on the left side with midline extension of $8 \mathrm{~mm}$ to right with involvement of genioglossus bilaterally and left valecula - Carcinoma tongue

trigone.

Postoperative HPE findings correlated well with imaging diagnosis in cases of malignant lesions. Only one case was proven to be a thyroglossal duct cyst. Vascular lesions were managed with a combination of clinical and imaging findings $\&$ embolization was done where ever necessary. Other benign lesions were asked for monitoring with follow-up after six months without intervention.

\section{Discussion}

\section{Normal tongue Anatomy}

The oral cavity includes the lips anteriorly; circumvallate papillae, tonsillar pillars, and soft palate posteriorly; mandibular alveolar ridge, mylohyoid muscles, and the teeth inferiorly, gingivobuccal region laterally; hard palate, maxillary alveolar ridge and the teeth superiorly. ${ }^{[5]}$ The terms' floor of the mouth' and 'root of the tongue' refer to the oral cavity, whereas the

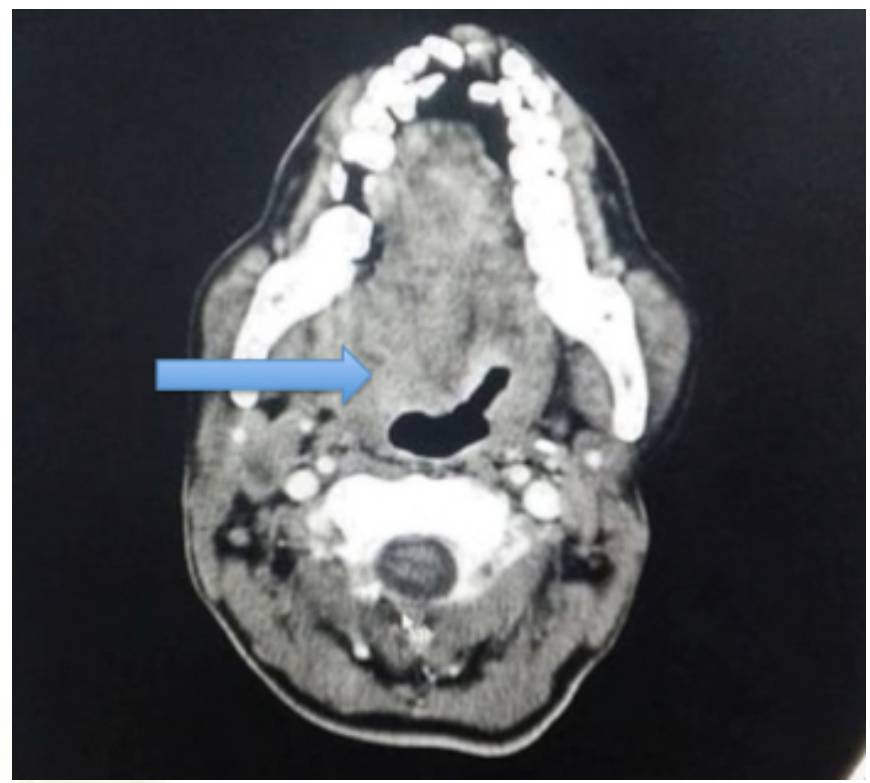

Figure 3: Contrast enhanced axial CT image of a 40 year old female patient shows an ill-defined heterogeneously enhancing lesion in the right half of the tongue involving the intrinsic muscles both anteriorly and posteriorly - Carcinoma tongue. $-\mathrm{NAD} \bullet$ Hemangioma $\bullet$ Lipoma $\bullet$ TG cyst $\bullet$ Venous maltormations
SCC

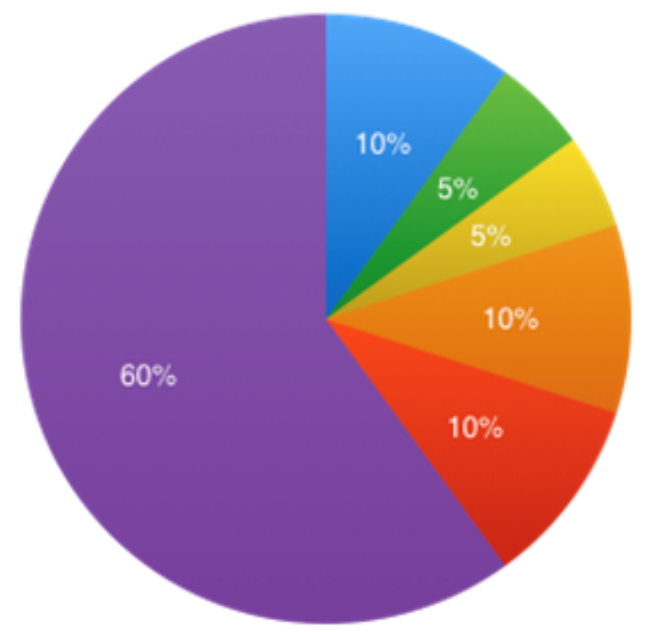

Graph 1: Depict the percentage of various lesions seen in our patients

term 'base of the tongue' pertains to a region in the oropharynx. The superior and inferior longitudinal, transverse, vertical, and oblique muscles constitute the intrinsic muscles of the tongue. Muscles that allow attachment of the tongue to 


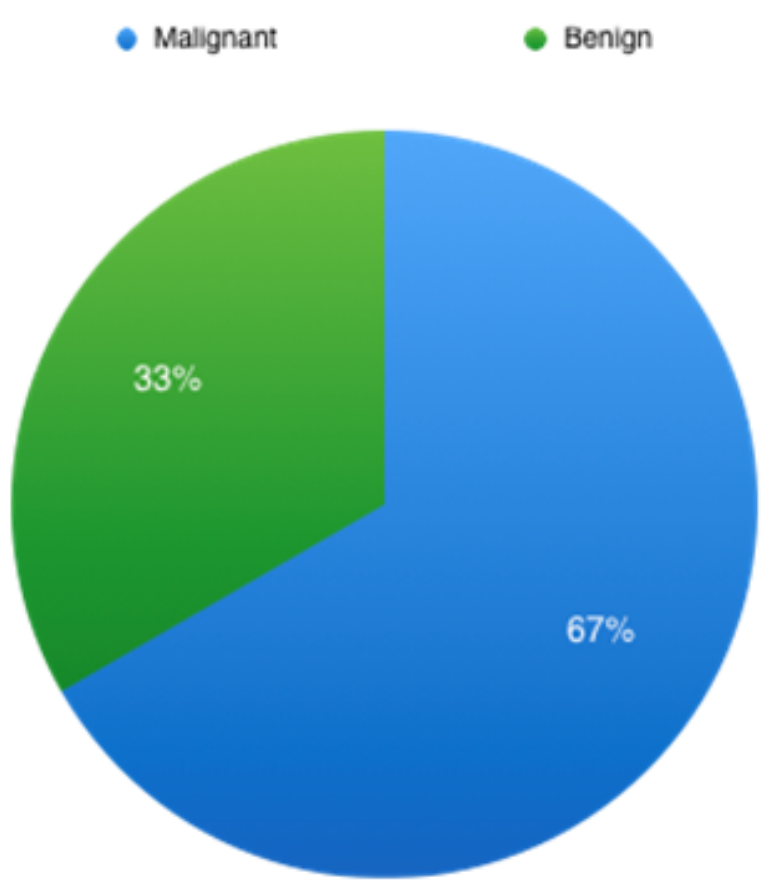

Graph 2: Depicts the percentage of patients with benign and malignant lesions

hyoid bone, mandible, and styloid process of the skull base are the extrinsic muscles, which include genioglossus, hyoglossus, palatoglossus, and styloglossus. The hypoglossal nerve traversing between the mylohyoid and hyoglossus tissue innervates all the muscles of the tongue except palatoglossus, which is supplied by pharyngeal plexus. The lingual nerve, which courses adjacent to the hypoglossal nerve, is the sensory supply to the anterior two-thirds of the tongue. Glossopharyngeal nerve supplies posterior one-third of the tongue. Individual sensory fibers for taste course along the lingual nerve and join forming the chords tympani nerve, which traverses the middle ear and joins the facial nerve. ${ }^{[6]}$

\section{Imaging Features of various lesions involving the tongue}

Lesions arising from the root of the tongue can be classified into congenital (vascular and nonvascular lesions), infections, and neoplasms. The largest group of injuries comprise of the congenital vascular and nonvascular lesions. In contrast, lesions occurring much more frequently in the adjacent sublingual and submandibular spaces, and the base of the tongue are of acquired type. The higher prevalence of acquired lesions is presumably due to the greater exposure to the mucosal surfaces and prominent lymphoid tissues. Also, the skeletal muscle that composes most of the root of the tongue is less prone to neoplasms and relatively resistant to infection as compared with other tissues. ${ }^{[7]}$

\section{Lingual Thyroid}

Ectopic thyroid tissues are found along the thyroglossal duct, which is situated between the foramen cecum and the thyroid gland during development, and involute in adults. Before it reaches the hyoid bone, the canal passes through the posterior aspect of the root of the tongue. The thyroglossal duct is found along the midline in the suprahyoid neck. However, in the infrahyoid neck, it diverges laterally. ${ }^{[8]}$ On CT, lingual thyroids appear characteristically hyperattenuating relative to muscle due to, (a) The iodine content of thyroid tissue and

(b) Moderate contrast enhancement on post-contrast images. On MRI, ectopic thyroid usually appears solid, mildly T1 hyperintense or isointense relative to muscle, and avidly enhancing. The uptake of high iodine-123 (123I) and technetium-99m on radiotracer studies is very specific. Similar to other thyroid tissues, these lesions can undergo goitrous and malignant transformation ( $3 \%$ of cases).

\section{Thyroglossal Duct Cyst}

Similar to ectopic thyroids, thyroglossal duct cysts are seen along the thyroglossal duct. They account for the most common thyroglossal duct lesions, wherein approximately $20 \%-25 \%$ are suprahyoid in location. These lesions are characteristically cystic in appearance, sometimes showing thin septae or lobulations. Like most of the cystic lesions, thyroglossal duct cysts show an attenuation value that is usually between 0 and $20 \mathrm{HU}$ at $\mathrm{CT}$, with hyperintensity on $\mathrm{T} 2$, and intermediate $\mathrm{T} 1$ signal at MR imaging. The contents within the lesion can be proteinaceous or hemorrhagic, making the lesion hyperdense on $\mathrm{CT}$, and hyperintense at T1 sequence. Most of the lesions are well- circumscribed, showing a very thin rim enhancement. Extensive surrounding soft-tissue edema with heterogeneous and complex appearance may be seen with lesions that are infected or hemorrhagic. When a component closely associated with the hyoid bone is discovered, thyroglossal duct cysts may be distinguished from other cystic lesions. ${ }^{[9]}$

\section{Dermoid and Epidermoid Cysts}

Dermoid and epidermoid cysts in the oral cavity are most commonly found at the floor of the mouth and the root of the tongue. The distinction between dermoid and epidermoid cysts at imaging may be challenging as both the lesions are well-circumscribed and exhibit high T2 signal with no enhancement or only rim enhancement. Epidermoid cysts have only epithelial elements, whereas dermoid cysts contain both epithelial components and a dermal substructure. At imaging, significant signal heterogeneity (i.e., from the combination of solid and cystic factors) reflects the additional complexity of dermoid cysts. Epidermoid cysts usually do not exhibit substantial solid components. Intralesional fat is a distinguishing feature in dermoid cysts. A nearly pathognomonic appearance of a "sack of marbles" may 
be created when this fat coalesce into globules. ${ }^{[10]}$ At diffusion imaging, epidermoid cysts may show restriction (high diffusion and a low apparent diffusion coefficient). However, the visible diffusion coefficient values are typically only moderately low and are a characteristic feature.

\section{Lipoma}

Lipomas are usually easily identified, well-encapsulated lesions with the attenuation or signal intensity of fat. Lipomas account for only $0.1 \%-5 \%$ of benign lesions in the oral cavity, but $50 \%$ of these localize to the buccal soft tissues.

\section{Foregut Duplication Cyst}

Foregut duplication cysts are seldom seen in the root of the tongue. These cysts usually exhibit a CT attenuation of that of fluid but may vary depending on proteinaceous content. At MR imaging, these cysts show a high T2 signal and a T1 signal that varies depending on proteinaceous content. Like thyroglossal duct cysts, they have a uniform enhancing rim and can have thin septa or lobulations. Foregut duplication cysts may also become infected or hemorrhage, leading to a heterogeneous appearance.

\section{Vascular malformations}

Based on the growth and histological differences, vascular malformations are divided into hemangioma and vascular malformations. Vascular malformations are further subcategorized into high flow lesions like arteriovenous malformations and low flow lesions, such as lymphatic and venous malformation. Of these, soft tissue masses presenting at birth are relatively more contributed by lymphatic and venous malformations. ${ }^{[11]}$ Low flow venous malformations are characterized by $\mathrm{T} 2$ hyperintense venous lakes with flow voids within, which represent phleboliths. Lymphatic malformations may be of two types, namely microcystic or macro cystic malformations. Macro-cystic type is T2 hyperintense and maybe uni /multilocular cystic mass with fluid level within. But microcystic malformation appears as an area of high signal intensity on T2-weighted imaging. Angiography is usually used to confirm high flow malformations, which show abnormal arterial supply to the tongue with a abnormal prominent vascular blush. Hemangioma exhibits strong signals on T1-weighted imaging, heterogeneous high signal on T2-weighted imaging, and prominent enhancement with a lack of signal voids. Involuting hemangioma shows high $\mathrm{T} 1$ signals representing fatty replacement.

\section{Squamous cell carcinoma}

Squamous cell carcinoma (SCC) of the oral cavity has a predilection for the gingivo-buccal region, tongue, and retromolar trigone. The second most common site for SCC of the oral cavity is the tongue. The prevalence of SCC has been rising in India and the west due to excessive abuse of tobacco and alcohol. SCC usually presents clinically as ulcers, which can be diagnosed with ease by biopsy. Staging the lesion is the primary concern of imaging.

Staging for the SCC of the oral cavity is as follows; T0no evidence of a primary tumor; T1- greatest diameter of the primary tumor is less than $2 \mathrm{~cm}$; T2-greater than $2 \mathrm{~cm}$ but less than $4 \mathrm{~cm}$ in diameter; T3- primary tumor greater than $4 \mathrm{~cm}$; T4-a massive tumor more than $4 \mathrm{~cm}$ in diameter with deep invasion, involving the antrum, pterygoid muscles, base of tongue or skin of the neck. The above-mentioned staging system is used by The American Joint Committee on Cancer and the International Union against Cancer (UICC). As thickness greater than $4 \mathrm{~mm}$ has been associated with cervical nodal metastasis, assessing tumor thickness is essential in the early stages. ${ }^{[12]}$

The precise measurement for assessing the tumors from the lateral border of the tongue is the lateromedial thickness taken in the axial MR plane. Another study by Okura et al. established a significant predictor in a tumor with depth $>9.7$ $\mathrm{mm}$ for nodal metastasis. ${ }^{[13]}$

The other predictors for staging the tumor is the assessment of the involvement of muscles, with or without crossing of midline, extension into the floor of mouth, valleculae, pre- epiglottic space, and the hyoid bone. The involvement of vallecular, pre-epiglottic space and hyoid bone indicate relative contraindications for surgical resection.

SCC commonly involves the level I and level II neck nodes. Skip metastasis to level III, IV, contralateral level I and II lymph nodes are also noted. Metastatic lymph nodes appear enlarged, round and show necrosis. Circumferential contact of greater than 270 degrees of the lymph nodes with the carotid artery precludes the resectability of the node.

An increase in tumor size causes an increase in heterogeneity of tumors which also indicates the degree of necrosis. Cortical bone invasion is indicated by erosions of the bone. A hyperdense area replacing the healthy fat in CT denotes medullary bone involvement. ${ }^{[14]}$

Non-contrast $\mathrm{T} 1$ weighted images yield satisfactory details on cortical erosion and bone marrow invasion. However, contrastenhanced T1 weighted imaging aids in the assessment of marrow invasion, perineural spread, soft tissue extent, tumor thickness, and necrotic lymph nodes. ${ }^{[15]} \mathrm{T} 2$ weighted imaging provides useful information regarding the involvement of extrinsic muscle and floor of the mouth. STIR and DWI sequences are of great importance in visualizing lymph nodes, whereas the latter is an added advantage in assessing subcentimetric lymph nodes.

\section{Conclusion}

Few specific lesion characteristics can aid in narrowing the differential diagnosis. Solid high-density lesions in the midline 
mostly represent lingual thyroids. These lesions may be verified with nuclear imaging. Calcifications likely indicate goitrous transformation. Phleboliths are highly suggestive of venous malformations. Multilocular, thin-rim enhancing cystic lesions are indicative of lymphatic malformations, primarily when fluid-fluid levels are found. Fat/calcium content within a complex cystic lesion is specific for a dermoid cyst, whereas diffusion restriction within a single cystic lesion is suggestive of an epidermoid cyst. Finally, when a lesion is Trans spatial, three differentials to be considered are - highly aggressive malignancies, congenital masses \& aggressive infections. Although MRI is a more sensitive modality, CT is the most commonly used investigation for pre-operative planning \& postoperative follow-up. Plain CT is useful in assessing the involvement of adjacent bones, while MRI is helpful in identifying the flow voids, the extent of soft tissue involvement \& neurovascular bundle assessment.

\section{References}

1. Badar Z, Farooq Z, Zaccarini D, Ezhapilli SR. Tongue base schwannoma: differential diagnosis and imaging features with a case presentation. Radiol Case Rep. 2016;11(4):336340. Available from: https://dx.doi.org/10.1016/j.radcr.2016. 10.001

2. Sankaranarayan R, Masuyer E, Swaminathan R, Ferley J, Whelan S. Head and neck cancer: A global perspective on epidemiology and prognosis. Anticancer Res. 1998;18:477986.

3. Sigal R, Zagdanski AM, Schwaab G, Bosq J, Auperin A, Laplanche A, et al. CT and MR imaging of squamous cell carcinoma of the tongue and floor of the mouth. RadioGraphics. 1996;16(4):787-810. Available from: https://dx.doi.org/10. 1148/radiographics.16.4.8835972.

4. II RKH, Strain JD. MRI-compatible audio/visual system: impact on pediatric sedation. Pediatric Radiology. 2001;31(4):247-250. Available from: https://dx.doi.org/10. $1007 / \mathrm{s} 002470100426$.

5. Subramanian K, Ramachandran R, Chandrasekharan A, Rangasami R, M VSP, Joseph S. Imaging in benign and malignant mass lesions of the tongue. IJAM. 2016;3(1):57-62. Available from: http://dx.doi.org/10.18203/2349-3933.ijam20160189.

6. Meesa IR, Srinivasan A. Imaging of the Oral Cavity. Radiologic Clin North Am. 2015;53(1):99-114. Available from: https://dx.doi.org/10.1016/j.rcl.2014.09.003.

7. Ozturk M, Mavili E, Erdogan N, Cagli S, Guney E. Tongue abscesses: MR imaging findings. Am J Neuroradiol.
2006;27(6):1300-1303.

8. Patel S, Bhatt AA. Thyroglossal duct pathology and mimics. Insights Imaging. 2019;10:1-12. Available from: https://dx. doi.org/10.1186/s13244-019-0694-x.

9. Chandra RK, Maddalozzo J, Kovarik P. Histological Characterization of the Thyroglossal Tract: Implications for Surgical Management. Laryngoscope. 2001;111(6):10021005. Available from: https://dx.doi.org/10.1097/00005537200106000-00014.

10. Koeller KK, Alamo L, Adair CF, Smirniotopoulos JG. From the Archives of the AFIP. RadioGraphics. 1999;19(1):121-146. Available from: https://dx.doi.org/10.1148/radiographics.19.1. g99ja06121.

11. Donnelly LF, Jones BV, L J. Imaging of pediatric tongue abnormalities. Am J Roentgenol . 2000;175(2):48993-48995. Available from: https://doi.org/10.2214/ajr.175.2.1750489.

12. Huang SH, Hwang D, Lockwood G, Goldstein DP, O'Sullivan B. Predictive value of tumor thickness for cervical lymph-node involvement in squamous cell carcinoma of the oral cavity. Cancer. 2009;115(7):1489-1497. Available from: https://dx. doi.org/10.1002/cncr.24161.

13. Arakawa A, Tsuruta J, Nishimura R, Sakamoto Y, Korogi Y, Baba Y. MR imaging of lingual carcinoma: Comparison with surgical staging. Radiat Med. 1996;14:25-29.

14. Chung TS, Yousem DM, Seigerman HM, Schalkman BN, Weinstein GS, Hayden RE. MR of mandibular invasion in patients with oral and oropharyngeal malignant neoplasms. Am J Neuroradiol. 1994;15:1949-1955.

15. Imaizumi A, Yoshino N, Yamada I, Nagumo K, Amagasa T, Omura K. A potential pitfall of MR imaging for assessing mandibular invasion of squamous cell carcinoma in the oral cavity. Am J Neuroradiol. 2006;27:114-122.

Copyright: (C) the author(s), 2020. It is an open-access article distributed under the terms of the Creative Commons Attribution License (CC BY 4.0), which permits authors to retain ownership of the copyright for their content, and allow anyone to download, reuse, reprint, modify, distribute and/or copy the content as long as the original authors and source are cited.

How to cite this article: Deepthi BT, Gautham M, Mathew JR. Role of Cross-Sectional Imaging in Tongue Lesions. Asian J. Med. Radiol. Res. 2020;8(1):58-63.

DOI: dx.doi.org/10.47009/ajmrr.2020.8.1.12

Source of Support: Nil, Conflict of Interest: None declared. 